\title{
mRNA expression profiling of mitochondrial subunits in subjects with Parkinson's disease
}

\author{
Michele Salemi ${ }^{1}$, Filomena Cosentino ${ }^{1}$, Giuseppe Lanza ${ }^{1,2}$, Mariagiovanna Cantone ${ }^{3}$, \\ Maria Grazia Salluzzo ${ }^{1}$, Giorgio Giurato ${ }^{4}$, Eugenia Borgione ${ }^{1}$, Giovanna Marchese ${ }^{5}$, \\ Sandro Santa Paola ${ }^{1}$, Bartolo Lanuzza ${ }^{1}$, Corrado Romano ${ }^{1}$, Raffaele Ferri ${ }^{1}$
}

${ }^{1}$ Oasi Research Institute-IRCCS, Troina, Italy

${ }^{2}$ Department of Surgery and Medical-Surgical Specialties, University of Catania, Catania, Italy

${ }^{3}$ Department of Neurology, Sant'Elia Hospital, Azienda Sanitaria Provinciale (ASP) Caltanissetta, Caltanissetta, Italy

${ }^{4}$ Laboratory of Molecular Medicine and Genomics, Department of Medicine, Surgery and Dentistry 'Scuola Medica Salernitana', University of Salerno, Salerno, Italy

${ }^{5}$ Genomix4Life Srl, Baronissi (SA) 84081, Italy

Submitted: 8 September 2020; Accepted: 13 December 2020

Online publication: 28 March 2021

Arch Med Sci

DOI: https://doi.org/10.5114/aoms/131629

Copyright $\odot 2021$ Termedia \& Banach

\begin{abstract}
Introduction: Parkinson's disease (PD) is a common adult-onset neurodegenerative disorder caused by a progressive loss of dopaminergic neurons due to the accumulation of $\alpha$-synuclein in the substantia nigra. Mitochondria are known to play a key role in cell respiratory function and bioenergetics. Indeed, mitochondrial dysfunction causes insufficient energy production required to satisfy the needs of several organs, especially the nervous system. However, the profiling of messenger RNA (mRNA) expression of mitochondrial subunits in PD has not been systematically investigated yet.

Material and methods: We explored the mRNA expression of mitochondrial DNA (mtDNA) encoded respiratory chain (RC) subunits in 43 PD patients and 43 normal controls (NC). Next generation sequencing analysis (NGS) was used and quantitative real-time polymerase chain reaction (qRT-PCR) assay was used for confirmation of the NGS results.

Results: All tested mitochondrial RC subunits were significantly over-expressed in subjects with PD compared to NC. In qRT-PCR the mean expression of all mitochondrial subunits had an expression level of at least 7 times compared to NC.

Conclusions: The over-expression of mitochondrial subunits in PD subjects with respect to NC might be secondary to a degeneration-related alteration of the mitochondrial structure and/or dynamics, or to the occurrence of a compensatory mechanism. The study of specific mRNA by peripheral blood mononuclear cells may provide a further diagnostic frame for early detection PD patients.
\end{abstract}

Key words: Parkinson's disease, mitochondrial subunits, NGS, qRT-PCR.

\section{Introduction}

Parkinson's disease (PD) is the most common neurodegenerative disorder after Alzheimer's disease (AD). Parkinson's disease typically develops between the ages of 55 and 65 years. Approximately $2 \%$ of people over the age of 60 years are affected, and this percentage rises to 3.5\% at the age of 85-89 years [1].

\section{Corresponding author:}

Dr. Michele Salemi

Oasi Research Institute-IRCCS

Via Conte Ruggero

78 - 94018, Troina, Italy

Tel.: + 390935936440

Fax: +390935936593

E-mail: micezia@tiscali.it 
M. Salemi, F. Cosentino, G. Lanza, M. Cantone, M. Grazia Salluzzo, G. Giurato, E. Borgione, G. Marchese, S. Santa Paola, B. Lanuzza, C. Romano, R. Ferri

Parkinson's disease is a complex neurological disease associated with the progressive degeneration of dopaminergic neurons in the substantia nigra pars compacta (SNPC) and in the locus coeruleus. The histopathological hallmarks of PD are the accumulation of aggregates of $\alpha$-synuclein, known as Lewy bodies, in the SNPC, causing degeneration of the extrapyramidal circuits of the midbrain and basal ganglia, eventually resulting in several signs and symptoms due to the impairment of motor control and modulation [2, 3]. The intracellular aggregates of $\alpha$-synuclein damage the neuronal membrane, inducing the phenomena of oxidative stress, excitotoxicity, energy failure, and neuroinflammation, thus leading to neural death [4].

Both motor and non-motor signs characterize the clinical picture of typical PD patients. Among the former, bradykinesia, resting tremor, muscle rigidity, postural instability, and hypomimic face are common manifestations [5]. The accumulation of Lewy bodies also in the cerebral cortex and autonomic and peripheral nervous system [6] produces a variety of non-motor symptoms, such as cognitive decline until dementia, mood changes, sleep dis- orders, and dysautonomia (including hypotension and urinary disturbances). Cognitively, sequence learning, motor sequence, switching between different tasks, and verbal fluency are the cognitive domains usually affected in PD patients [7].

In this scenario, PD pathophysiology also includes mitochondrial dysfunction and the related inhibition of the electron transport chain. These pathomechanisms lead to the generation of reactive oxygen species (ROS) and cellular energy failure, thus consequently causing cellular damage and neuronal death.

Mitochondria are present in all nucleated human cells and underlie multiple functions, especially the generation of energy for the cell. Mitochondrial dysfunction leads to insufficient energy production required to satisfy the needs of several organs, especially the nervous system [8]. Growing evidence has shown that impairment of the mitochondrial respiratory chain plays a key role in several neurodegenerative diseases, including PD [8, 9].

Specific elements of the respiratory chain within the mitochondrial membrane can be impaired following a disruption of the electron transport efficiency. Namely, defects of complex I, which

Table I. Description of the studied mitochondrial subunits

\begin{tabular}{|c|c|c|c|c|c|c|}
\hline Gene ID & Symbol & Aliases & $\begin{array}{l}\text { Descrip- } \\
\text { tion }\end{array}$ & Chromosome & $\begin{array}{l}\text { genomic_- } \\
\text { nucleotide_- } \\
\text { accession. } \\
\text { version }\end{array}$ & $\begin{array}{l}\text { start_posi- } \\
\text { tion_end_ } \\
\text { position_ }\end{array}$ \\
\hline 4512 MT-CO1 & $\begin{array}{c}\text { COI, } \\
\text { MTCO1, } \\
\text { COX1 }\end{array}$ & $\begin{array}{l}\text { mitochondrially encoded } \\
\text { cytochrome c oxidase I }\end{array}$ & MT & NC_012920.1 & 5904 & 7445 \\
\hline 4535 MT-ND1 & $\begin{array}{l}\text { MTND1, } \\
\text { ND1 }\end{array}$ & $\begin{array}{l}\text { mitochondrially encoded } \\
\mathrm{NADH} \text { dehydrogenase } 1\end{array}$ & MT & NC_012920.1 & 3307 & 4262 \\
\hline 4538 MT-ND4 & $\begin{array}{l}\text { MTND4, } \\
\text { ND4 }\end{array}$ & $\begin{array}{l}\text { mitochondrially encoded } \\
\mathrm{NADH} \text { dehydrogenase } 4\end{array}$ & MT & NC_012920.1 & 10760 & 12137 \\
\hline 4540 MT-ND5 & $\begin{array}{l}\text { MTND5, } \\
\text { ND5 }\end{array}$ & $\begin{array}{l}\text { mitochondrially encoded } \\
\mathrm{NADH} \text { dehydrogenase } 5\end{array}$ & MT & NC_012920.1 & 12337 & 14148 \\
\hline 4508 MT-ATP6 & $\begin{array}{c}\text { ATPase6, } \\
\text { MTATP6, } \\
\text { ATP6 }\end{array}$ & $\begin{array}{l}\text { mitochondrially encoded } \\
\text { ATP synthase } 6\end{array}$ & MT & NC_012920.1 & 8527 & 9207 \\
\hline 4537 MT-ND3 & $\begin{array}{l}\text { MTND3, } \\
\text { ND3 }\end{array}$ & $\begin{array}{l}\text { mitochondrially encoded } \\
\mathrm{NADH} \text { dehydrogenase } 3\end{array}$ & MT & NC_012920.1 & 10059 & 10404 \\
\hline 4541 MT-ND6 & $\begin{array}{l}\text { MTND6, } \\
\text { ND6 }\end{array}$ & $\begin{array}{l}\text { mitochondrially encoded } \\
\mathrm{NADH} \text { dehydrogenase } 6\end{array}$ & MT & NC_012920.1 & 14149 & 14673 \\
\hline 4513 MT-CO2 & $\begin{array}{c}\text { COII, } \\
\text { MTCO2, } \\
\text { cox2 }\end{array}$ & $\begin{array}{l}\text { mitochondrially encoded } \\
\text { cytochrome c oxidase II }\end{array}$ & MT & NC_012920.1 & 7586 & 8269 \\
\hline 4509 MT-ATP8 & $\begin{array}{c}\text { ATPase8, } \\
\text { MTATP8, } \\
\text { ATP8 }\end{array}$ & $\begin{array}{l}\text { mitochondrially encoded } \\
\text { ATP synthase } 8\end{array}$ & MT & NC_012920.1 & 8366 & 8572 \\
\hline 4514 MT-CO3 & $\begin{array}{l}\text { COIII, } \\
\text { MTC03, } \\
\text { cox3 }\end{array}$ & $\begin{array}{l}\text { mitochondrially encoded } \\
\text { cytochrome c oxidase III }\end{array}$ & MT & NC_012920.1 & 9207 & 9990 \\
\hline 4536 MT-ND2 & $\begin{array}{l}\text { MTND2, } \\
\text { ND2 }\end{array}$ & $\begin{array}{l}\text { mitochondrially encoded } \\
\mathrm{NADH} \text { dehydrogenase } 2\end{array}$ & MT & NC_012920.1 & 4470 & 5511 \\
\hline
\end{tabular}


represents the first step in the electron transport chain of mitochondrial oxidative phosphorylation (OXPHOS), have been documented in SNPC, platelets, and skeletal muscle of idiopathic PD patients [10-12]. Since then, altered complex I activity has been reported in fibroblasts and in the frontal cortex $[13,14]$. Complex IV (or cytochrome c oxidase) represents the last enzyme of this chain, whereas complex $V$ (ATP synthase) is a ubiquitarian membrane enzyme playing a crucial role in energy metabolism [15].

In this context, mitochondrial dysfunction has been closely linked to the development and pathophysiology of PD [16]. In particular, damage of the electron transport chain, inhibition of complex I activity, mishandling of calcium, enhanced sensitivity to mitochondrial toxins, oxidative stress, and dysfunctional mitochondrial dynamics might all contribute to neuronal dysfunction and take part in the onset, course, and severity of PD [17]. As the relevant energy-producing organelles within the cell, mitochondria oxidize carbon and further release adenosine triphosphate (ATP) through oxidative phosphorylation [18]. Furthermore, progressive mitochondrial damage might lead to the accumulation of non-functional mitochondria, thus further contributing to cellular dysfunction and neuronal degeneration [17].

Based on these considerations, in the present study we evaluated the expression of the mRNA of eleven mitochondrial subunits (Table I) in a sample of PD patients compared to normal controls (NC). We hypothesized that specific mRNA expression profiling of mitochondrial subunits might be useful for early detection of PD and differentiation with NC.

\section{Material and methods}

\section{Participants and samples for qRT-PCR and RNA sequencing study}

For the analysis through qRT-PCR, a total of 86 participants, including 43 NC (15 females and 28 males; age range 56-84 years) and 43 PD patients (15 females and 28 males; age range $56-84$ years) were enrolled at the Oasi Research Institute-IRCCS, Troina (Italy). Both NC and PD patients were Caucasian and of Sicilian ancestry. Family history was obtained through a detailed interview with a first-degree relative or the proband spouse. Clinical and past medical history of each patient was collected, including drug prescriptions, medical records, and medical certificates. Clinical diagnoses were provided by a trained neurologist according to the current diagnostic criteria for PD [19].

For the analysis through next generation sequencing (NGS), 16 NC (9 females and 7 males; age range $50-88$ years) and 21 PD patients (8 fe- males and 13 males; age range $51-86$ years) were recruited at the Oasi Research Institute-IRCCS, Troina (Italy). Blood samples were obtained from each participant, as described by Salemi et al. (2018) [20].

Informed consent for inclusion in the study was obtained from all subjects or their legal guardians. The study was carried out in accordance with the Declaration of Helsinki of 1964 and its later amendments, and the Ethics Committee of the Oasi Research Institute-IRCCS, Troina (Italy) approved the protocol on June 3, 2017 (2017/05/31/ CE-IRCCS-OASI/9).

\section{RNA extraction for qRT-PCR}

RNA extraction by peripheral blood mononuclear cells (PBMCS) and subsequent retro-transcription were performed using the RNeasy Mini Kit and QuantiTect Reverse Transcription Kit (Qiagen Sciences, Germantown, USA), as previously described [15]. For mRNA extraction, each whole blood sample (approximately $8 \mathrm{ml}$ ) was processed within 2 hours of collection and the mRNA was stored at $-80^{\circ} \mathrm{C}$. Two whole blood samples were processed for each patient.

The quantification of RNA was performed using the Nanodrop ND-1000 spectrophotometer (NanoDrop Technologies). The tracks of genomic DNA were removed using the QuantiTect Reverse Transcription Kit (Qiagen, Germany), with the thermocycler program: $2^{\prime}$ at $42^{\circ} \mathrm{C}$ with gDNA wipeout buffer. Complementary DNA (cDNA) synthesis was carried out using 100 ng of RNA and the QuantiTect Reverse Transcription Kit (Qiagen Sciences, Germantown, USA), thermocycler program: $15^{\prime}$ at $42^{\circ} \mathrm{C}$ and $3^{\prime}$ at $95^{\circ} \mathrm{C}$.

\section{RNA extraction for RNA sequencing}

PBMC separation was performed using Ficoll-Paque (Ficoll Plaque PLUS - GE Healthcare Life Sciences, Piscataway, USA) and the RNA was extracted using the TRIzol reagent (TRIzol Reagent, Invitrogen Life Technologies, Carlsbad, CA, USA), according to the instructions provided by the manufacturer. For mRNA extraction, each whole blood sample (approximately $10 \mathrm{ml}$ ) was processed within 2 hours of collection and the mRNA was stored at $-80^{\circ} \mathrm{C}$. One whole blood sample was processed for each patient.

\section{qRT-PCR}

In a total volume of $25 \mu \mathrm{l}$, the qRT-PCR experiments were performed using the Light Cycler 480 instrument (Roche Diagnostics; Mannheim, Germany). The mitochondrial subunits target gene assays (assay ID ATP6 Hs02596862_g1; assay ID ATP8 Hs02596863_g1; assay ID CO1 
Hs02596864_g1; assayIDCO2Hs02596865_g1; assay ID CO3 Hs02596866_g1; assay ID ND1 Hs02596873 s1; assay ID ND2 Hs02596874_g1; assay ID ND3 Hs02596875_s1; assay ID ND4 Hs02596876_g1; assayIDND5 Hs02596878_g1; assay ID ND6 Hs02596879_g1) [21] and the glyceraldehyde-3-phosphate dehydrogenase (GAPDH) reference gene assay (assay IDHs99999905_m1) were obtained from Applied Biosystems (Carlsbad, CA, USA).

The thermal cycling conditions consisted of one cycle of 2 min at $50^{\circ} \mathrm{C}$ (Uracil-DNA glycosylases incubation), one cycle of $15 \mathrm{~min}$ at $95^{\circ} \mathrm{C}$ (enzyme activation) and 42 cycles of 15 seconds at $94^{\circ} \mathrm{C}$, followed by $1 \mathrm{~min}$ at $60^{\circ} \mathrm{C}$ (PCR). The QuantiTect Probe PCR Kit (Qiagen Sciences, Germantown, USA) was used.

The amplified cDNA was quantified using the threshold cycle (Ct) method and the relative quantification analysis data were generated using the comparative $2^{-\Delta \Delta c t}$ method: each cDNA from PD subjects was coupled with the cDNA from NC. The Light Cycler 1.5 software package supplied with the Light Cycler 480 was used to analyze the relative quantification.

\section{Statistical analysis for qRT-PCR}

Distribution analysis of mitochondrial subunit levels was performed using the Shapiro-Wilk test, whereas the inferential statistical analysis was carried out using the Wilcoxon rank-sum test and bivariate linear regression analysis. Graph Pad Prism 5 software was used for statistical analysis. We considered a $p$-value $<0.05$ as statistically significant.

\section{RNA sequencing and data analysis}

In each sample, the RNA concentration was assayed with a ND-1000 spectrophotometer (NanoDrop) and its quality assessed with the TapeStation 4200 (Agilent Technologies). Indexed libraries were prepared from $1 \mu \mathrm{g} /$ ea purified RNA with TruSeq Stranded Total RNA (Illumina) Library Prep Kit, according to the manufacturer's instructions. Libraries were quantified using the Agilent 2100 Bioanalyzer (Agilent Technologies) and pooled such that each index-tagged sample was present in equimolar amounts, with a final concentration of the pooled samples of $2 \mathrm{nM}$. The pooled samples were subjected to cluster generation and sequencing using an Illumina NextSeq 500 System (Illumina) in a $2 \times 75$ paired-end format. The raw sequence files generated (fastq files) underwent quality control analysis using FastQC (https://www.bioinformatics.babraham.ac.uk/projects/fastqc/). Subsequently, the quality checked reads were trimmed with cutadapt v.1.10 and then aligned to the hu- man genome (hg38 assembly) using STAR v.2.5.2 [15], with standard parameters. The read-count for the genes of interest was computed using featureCounts [22] and normalized, considering all genes expressed in the samples, using DESeq2 [23]. RNA sequencing was performed by Genomix4Life srl (Baronissi, Italy). Raw RNA sequencing data are available in the NCBI SRA database (http://www. ncbi.nlm.nih.gov/bioproject/680218) with Accession Number PRJNA680218.

\section{Results}

NGS transcriptome analysis showed that the mitochondrial subunits MT-ATP6, MT-ATP8, MT-CO1, MT-CO2, MT-CO3, MT-ND1, MT-ND2, MT-ND3, MT-ND4, MT-ND5, and MT-ND6 were all upregulated in $\mathrm{PD}$ patients compared with $\mathrm{NC}$ (Figure 1). Except for the MT-CO1 $(p=0.07)$, all the other subunits were compared with NC with a $p<0.03$ and, in particular, a $p \leq 0.01$ was obtained for these subunits (Table II).

QRT-PCR analysis confirmed the NGS data. Accordingly, the mean expression of all mitochondrial subunits had an expression level of at least 7 times compared with NC (Figure 2). Statistical analysis showed a significant difference between PD and NC $(p<0.05)$ for all mitochondrial subunits, whereas no significant difference was found by analyzing subgroups by sex $(p>0.05)$.

To summarize, the results confirmed the data obtained by NGS analysis, and differences in values may reflect the methodological differences.

\section{Discussion}

The main finding of this study is an overall increase in mitochondrial gene expression in PD patients compared to NC. Given that all the mitochondrial subunits were found to be up-regulated, it is likely that mitochondrial complexes to which they belong might be considered to be impaired as well, probably suggesting a compensatory mechanism secondary to a deficient mitochondrial quantity or dynamics.

Mitochondria play a number of pivotal roles in respiratory functions and bioenergetics of the cell through several biochemical processes, such as the Krebs cycle and oxidative phosphorylation (OXPHOS) [24-26]. In this scenario, mitochondria continuously modulate their structure and functioning in response to the stimuli that the cell receives, the so-called "mitochondrial dynamics", in order to maintain proper cellular homeostasis. A reduction or lack of nutrients exposes mitochondria to elongation (fusion) in the attempt to increase the OXPHOS activity $[25,26]$, whereas an excess of nutrients induces mitochondrial fragmentation (fission) [24]. 
A

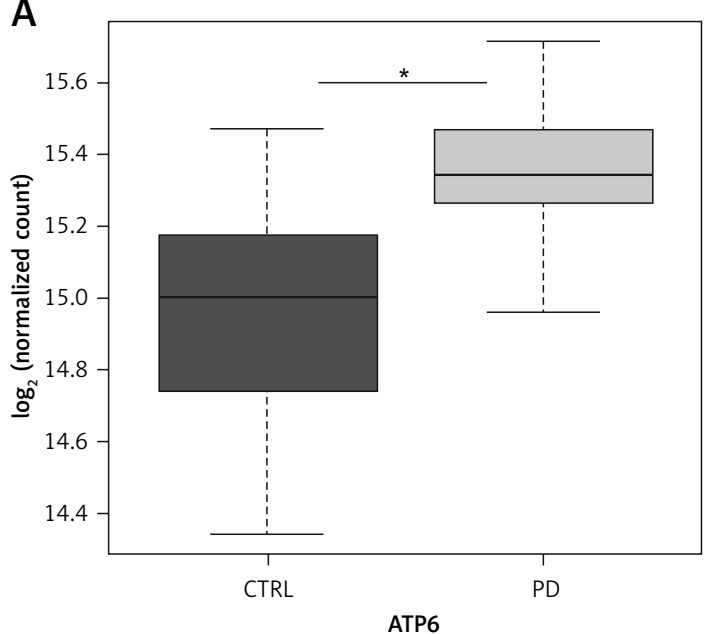

$\mathrm{C}_{176}$

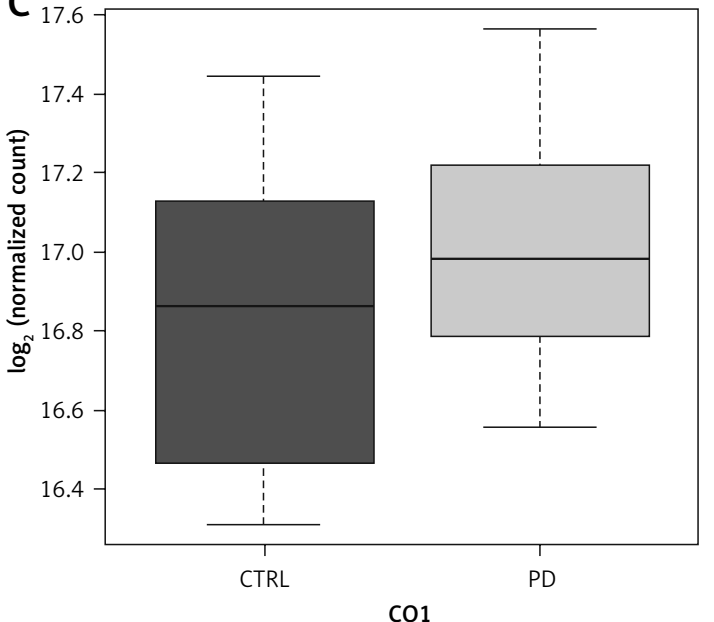

$\mathrm{E}$

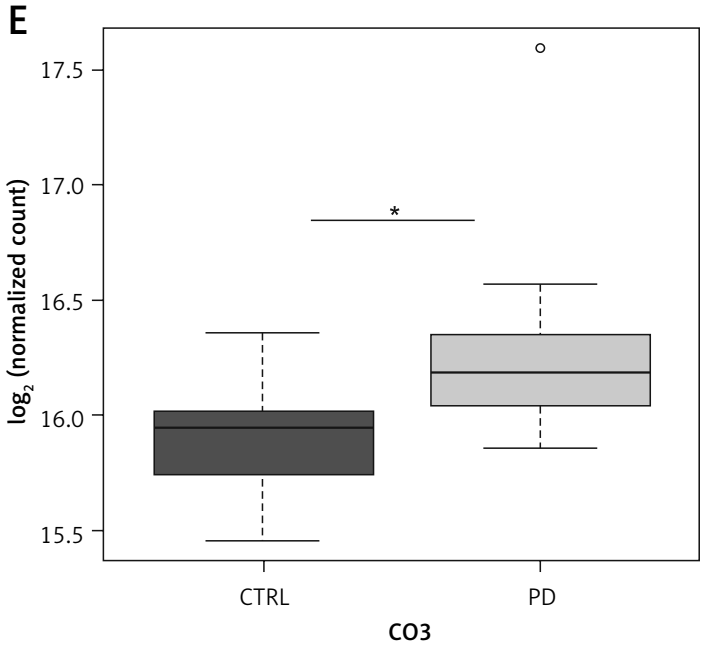

B

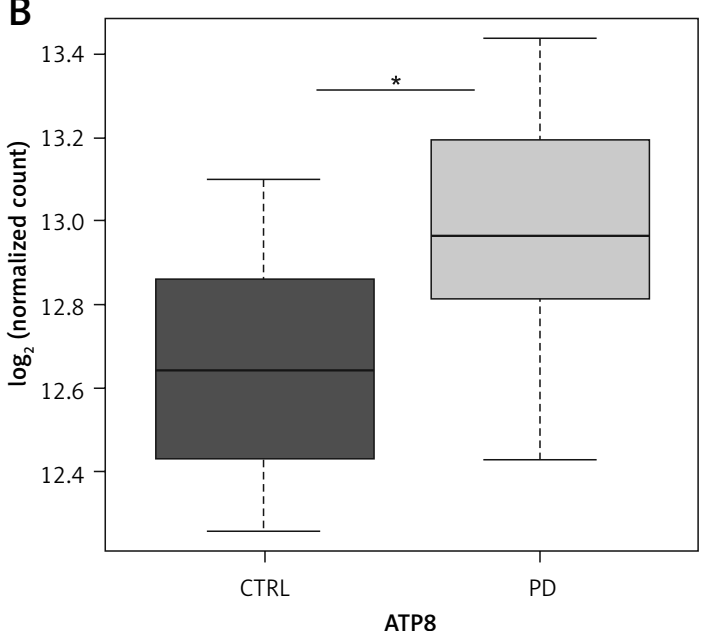

D

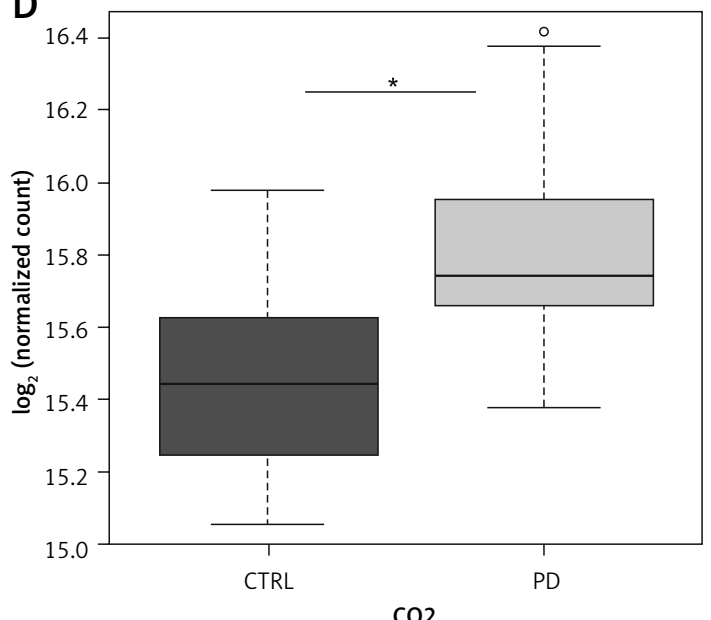

$\mathrm{F}$

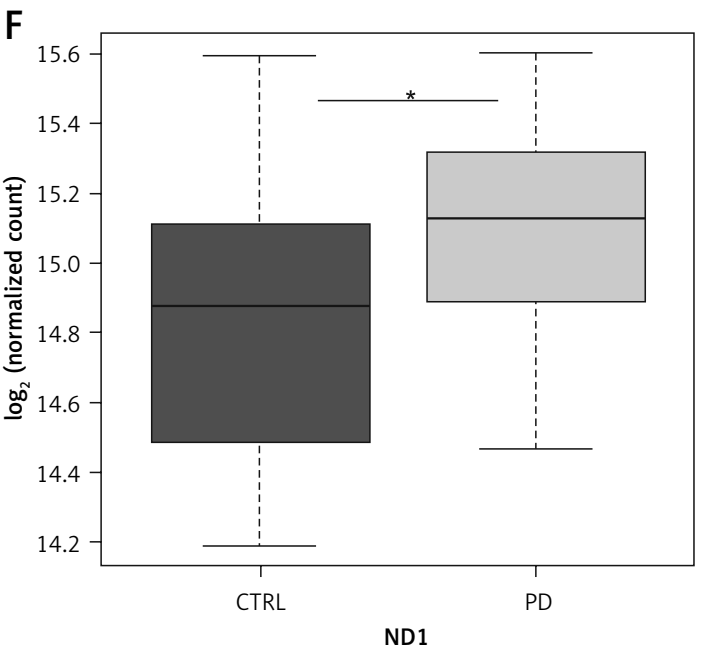

Figure 1. Box plots showing normalized expression values between the 15 samples of normal controls and 21 samples of PD patients, for each selected gene. ${ }^{*}=p<0.03$. Box plot of the distribution count for each sample. The frequency distribution, mean, median, and outliers are also presented. $\log _{2}$ normalized counts 
M. Salemi, F. Cosentino, G. Lanza, M. Cantone, M. Grazia Salluzzo, G. Giurato, E. Borgione, G. Marchese, S. Santa Paola, B. Lanuzza, C. Romano, R. Ferri
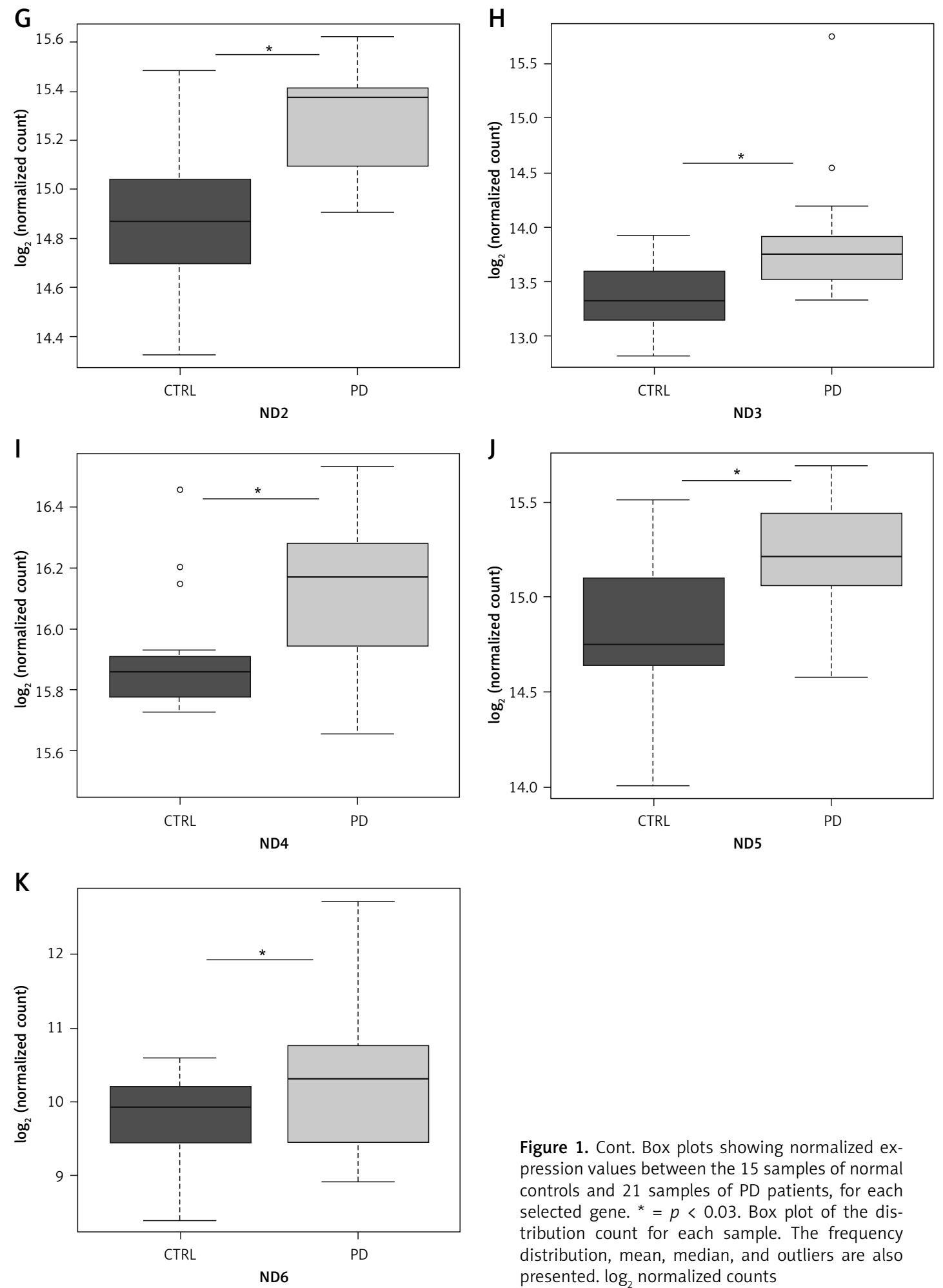

Mitochondrial dynamics also allows the compliance of mitochondrial activity with some physiological demands, such as $\mathrm{Ca}^{2+}$ homeostasis, ATP production, and regulation of ROS production [27]. Moreover, a selective autophagosomal process of mitochondrial degradation (mitophagy) to ensure quality control and cellular homeostasis [28] removes damaged mitochondria. Thus, a dysfunc-

Figure 1. Cont. Box plots showing normalized expression values between the 15 samples of normal controls and 21 samples of PD patients, for each selected gene. ${ }^{*}=p<0.03$. Box plot of the distribution count for each sample. The frequency distribution, mean, median, and outliers are also presented. $\log _{2}$ normalized counts

tion of mitochondrial dynamics or mitophagy is a key factor involved in the pathogenesis of different types of cancers, metabolic diseases, and neurodegenerative disorders $[29,30]$.

In PD, the impairment of mitochondrial dynamics exposes neuronal cells to a greater susceptibility to oxidative stress, eventually leading to mitochondrial fragmentation and fusion [31]. 
Table II. Difference of expression, along with $p$ value in normal controls and patients with Parkinson disease (PD)

\begin{tabular}{|lcccc|}
\hline Mitochondrial subunits & $p$-value & FC & Mean_CTRL & Mean_PD \\
\hline MT-ND6 & 0.02 & 1.747 & 927.916 & 1338.082 \\
\hline MT-ND1 & 0.029 & 1.181 & 30366.911 & 35914.986 \\
\hline MT-ATP6 & 0.0001087 & 1.287 & 32660.876 & 42581.551 \\
\hline MT-ND2 & $2.96 \mathrm{E}-05$ & 1.321 & 30833.090 & 41148.994 \\
\hline MT-ND3 & 0.01 & 1.505 & 10668.535 & 14514.746 \\
\hline MT-ND4 & 0.004 & 1.163 & 61605.741 & 73001.353 \\
\hline MT-CO2 & 0.0004059 & 1.282 & 45747.429 & 56915.571 \\
\hline MT-CO3 & 0.004072 & 1.317 & 61376.485 & 75332.634 \\
\hline MT-CO1 & 0.07 & 1.120 & 119436.076 & 136255.858 \\
\hline MT-ATP8 & 0.001 & 1.226 & 6623.219 & 8333.715 \\
\hline MT-ND5 & 0.002 & 1.281 & 30404.932 & 39995.435 \\
\hline
\end{tabular}

FC - fold change, mean CTRL - mean of normalized count in control $(n=15)$ samples, mean_PD - mean of normalized count in Parkinson disease $(n=21)$ samples.

Mitochondrial fusion compensates the functions of mitochondria through elongated tubules that facilitate the spread of various molecules throughout the mitochondrial compartments. Therefore, mitochondrial fusion is of crucial importance to avoid accumulation of damaged mitochondria, although excessive fusion of mitochondria inhibits the segregation and removal of damaged mitochondria themselves. Moreover, altered fusion inhibits the respiratory function in mitochondria, which, in turn, triggers excessive ROS production and leads to defective mitochondrial transports in dendrites or axons, with subsequent ATP deficiency in peripheral sites [32, 33-37].

Taken together, these considerations might explain the over-expression of mitochondrial mRNA subunits we observed in PD patients, possibly acting as a compensatory mechanism triggered by the alteration of mitochondrial structure and function. Conversely, the reduced expression of mitochondrial mRNA in dementia with Lewy bodies [38] suggests that the molecular mechanisms affecting mitochondrial structure and functioning may differ between PD and atypical parkinsonisms also at the subcellular level.

Environmental and food-related factors certainly correlate with the reduced risk for PD, including caffeine intake, and can positively affect the integrity of mtDNA by favoring a milder course of PD [39]. There is also evidence on how mitochondrial dysfunctions can affect neurodegenerative diseases other than PD, such as $A D$ $[40,41]$. These neurodegenerative conditions indeed show some similarities in the impairment of enzyme activities of complex I (to which the subunits MT-ND1, MT-ND2, MT-ND3, MT-ND4, MT-ND5 and MT-ND6 belong) and complex IV (to which the subunits MT-CO1, MT-CO2, MT-CO3 belong) at the level of the frontal cortex, substantia

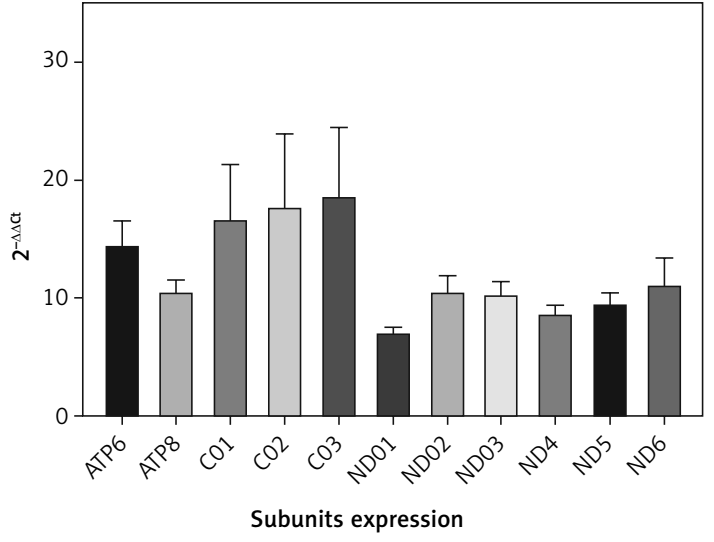

Figure 2. mRNA mitochondrial expression in 43 patients with Parkinson's disease and 43 normal controls (not included in the figure with value 1). Data were obtained by qRT-PCR, elaborated media mRNA levels by comparative $\Delta \Delta$ Ct method are shown

nigra, and cerebellum. These brain regions are all involved in patients with cognitive decline and in both motor and non-motor-related impairment of different neurotransmission systems [42]. In particular, complex I dysfunction seems to be critical in PD pathogenesis, because it correlates with the degeneration of dopaminergic neurons in the SNPC $[43,44]$.

The main limitation of this study is that the analysis of expression levels was performed on mRNA obtained from PBMCs. Given that neurons and blood cells have different environment and energetic demands, caution is needed when translating the results from blood cells to neurons. However, we cannot exclude that the mechanisms highlighted in PBMCs are not activated also in the brain (dopaminergic neurons) of the same subjects. In fact, as in other neurodegenerative diseases, a neurological impairment might 
be the result of a lack or a partial turnover of neurons.

In conclusion, our data showed overexpression of the mRNA mitochondrial subunits in PD patients, suggesting an alteration of the mitochondrial structure or the presence of compensatory mechanisms that would preserve the cell from programmed death (e.g. apoptosis due to the accumulation of ROS). The study of specific mRNA by PBMCs will provide further diagnostic frames for the early detection of PD patients. Future studies, also including patients with early-onset PD, are needed to validate this hypothesis and to expand the present findings to atypical parkinsonisms and other neurodegenerative diseases.

\section{Acknowledgement}

This study was supported by the Italian " $5 \mathrm{x}$ 1000" funding.

\section{Conflict of interest}

The authors declare no conflict of interest.

\section{References}

1. Rizek P, Kumar N, Jog MS. An update on the diagnosis and treatment of Parkinson disease. CMAJ 2016; 188 1157-65.

2. Cicero C.E, Mostile G, Vasta R, et al. Metals and neurodegenerative diseases. A systematic review. Environ Res 2017; 159: 82-94.

3. Politi C, Ciccacci C, Novelli G, Borgiani P. Genetics and treatment response in Parkinson's disease: an update on pharmacogenetic studies. Neuromol Med 2018; 20: 1-17.

4. Marques O, Outeiro TF. Alpha-synuclein: from secretion to dysfunction and death. Cell Death Dis 2012; 3: e350-7.

5. Sanjari Moghaddam H, Valitabar Z, Ashraf-Ganjouei A, Mojtahed Zadeh M, Ghazi Sherbaf F, Aarabi MH. Cerebrospinal fluid C-reactive protein in Parkinson's disease: associations with motor and non-motor symptoms. Neuromol Med 2018; 20: 376-85.

6. Schapira AHV. Glucocerebrosidase and Parkinson disease: recent advances. Mol Cell Neurosci 2015; 66 (Pt A): 37-42.

7. Stephan MA, Meier B, Zaugg SW, Kaelin-Lang A. Motor sequence learning performance in Parkinson's disease patients depends on the stage of disease. Brain Cogn 2011; 75: 135-40.

8. Lezi E, Swerdlow RH. Mitochondria in neurodegeneration. Adv Exp Med Biol 2012; 942: 269-86.

9. Power JH, Barnes OL, Chegini F. Lewy bodies and the mechanisms of neuronal cell death in Parkinson's disease and dementia with Lewy bodies. Brain Pathol 2015; 27: 3-12.

10. Parker WD, Boyson SJ, Parks JK. Abnormalities of the electron transport chain in idiopathic Parkinson's disease. Ann Neurol 1989; 26: 5.

11. Schapira AHV, Cooper JM, Dexter D, Jenner P, Clark JB, Marsden CD. Mitochondrial complex I deficiency in Parkinson's disease. Lancet 1989; 1: 1269.

12. Bindoff LA, Birch-Machin M, Cartlidge NEF, Parker WD, Turnbull DM. Mitochondrial function in Parkinson's disease. Lancet 1989: 2: 49.
13. Mytilineou C, Werner P, Molinari S, Di Rocco A, Cohen G, Yahr MD. Impaired oxidative decarboxylation of pyruvate in fibroblasts from patients with Parkinson's disease. J Neural Transm Park Dis Dement Sect 1994; 8 : 223-8.

14. Parker WD, Parksa JK, Swerdlow RH. Complex I deficiency in Parkinson's disease frontal cortex. Brain Res 2008; 1189: 215-8.

15. Houshmand M, Kasraie S, Ahari SE, Moin M, Bahar M, Zamani A. Investigation of tRNALys/Leu and ATPase $6 / 8$ gene mutations in Iranian ataxia telangiectasia patients. Arch Med Sci 2011; 7: 523-7.

16. Koyano F, Okatsu K, Ishigaki S, et al. The principal PINK1 and Parkin cellular events triggered in response to dissipation of mitochondrial membrane potential occur in primary neurons. Genes Cells 2013; 18: 672-81.

17. McCoy MK, Cookson MR. Mitochondrial quality control and dynamics in Parkinson's disease. Antioxid Redox Signal 2012; 16: 869-82.

18. Wallace DC, Fan W, Procaccio V. Mitochondrial energetics and therapeutics. Annu Rev Pathol 2010; 5: 297-348.

19. Postuma RB, Berg D, Stern M, et al. MDS clinical diagnostic criteria for Parkinson's disease. Mov Disord 2015 30: 1591-601.

20. Salemi M, Cimino L, Marino M, et al. Next generation sequencing expression profiling of mitochondrial subunits in men with Klinefelter syndrome. Int J Med Sci 2018; 15: 31-5.

21. Burgin HJ, Lopez Sanchez MIG, Smith CM, Trounce IA, McKenzie M. Pioglitazone and deoxyribonucleoside combination treatment increases mitochondrial respiratory capacity in m.3243A>G MELAS cybrid cells. Int J Mol Sci 2020; 21: 2139.

22. Liao Y, Smyth GK, Shi W. featureCounts: an efficient gen eral purpose program for assigning sequence reads to genomic features. Bioinformatics 2014; 30: 923-30.

23. Love MI, Huber W, Anders S. Moderated estimation of fold change and dispersion for RNA-seq data with DESeq2. Genome Biol 2014; 15: 550.

24. Youle RJ, van der Bliek AM. Mitochondrial fission, fusion, and stress. Science 2012; 337: 1062-5.

25. Amchenkova AA, Bakeeva LE, Chentsov YS, Skulachev VP, Zorov DB. Coupling membranes as energy-transmitting cables. I. Filamentous mitochondria in fibroblasts and mitochondrial clusters in cardiomyocytes. J Cell Biol 1988; 107: 481-95.

26. Skulachev VP. Mitochondrial filaments and clusters as intracellular power-transmitting cables. Trends Biochem Sci 2001; 26: 23-9.

27. Vafai SB, Mootha VK. Mitochondrial disorders as windows into an ancient organelle. Nature 2012; 491: 374-83.

28. Pickles S, Vigie P, Youle RJ. Mitophagy and quality control mechanisms in mitochondrial maintenance. Curr Biol 2018; 28: R170-R185.

29. Carelli V, Chan DC. Mitochondrial DNA: impacting central and peripheral nervous systems. Neuron 2014; 84 1126-42.

30. Lightowlers RN, Taylor RW, Turnbull DM. Mutations causing mitochondrial disease: what is new and what challenges remain? Science 2015; 349: 1494-9.

31. Kang TC. Nuclear factor-erythroid 2-related factor 2 (Nrf2) and mitochondrial dynamics/mitophagy in neurological diseases. Antioxidants (Basel) 2020; 9: E617.

32. Kim JE, Park H, Choi SH, Kong MJ, Kang TC. CDDO-Me selectively attenuates CA1 neuronal death induced by status epilepticus via facilitating mitochondrial fission independent of LONP1. Cells 2019; 8: 833. 
33. Twig G, Shirihai OS. The interplay between mitochondrial dynamics and mitophagy. Antioxid Redox Signal 2011; 14: 1939-51.

34. Kageyama Y, Zhang Z, Roda R, et al. Mitochondrial division ensures the survival of postmitotic neurons by suppressing oxidative damage. J. Cell Biol 2012; 197: 535-51.

35. Parone PA, Da Cruz S, Tondera D, et al. Preventing mitochondrial fission impairs mitochondrial function and leads to loss of mitochondrial DNA. PLoS One 2008; 3: e3257.

36. Kim JE, Ryu HJ, Kim MJ, Kang TC. LIM kinase-2 induces programmed necrotic neuronal death via dysfunction of DRP1-mediated mitochondrial fission. Cell Death Differ 2014; 21: 1036-49.

37. Kim JE, Choi HC, Song HK, Kang TC. Blockade of AMPA receptor regulates mitochondrial dynamics by modulating ERK1/2 and PP1/PP2A-mediated DRP1-S616 phosphorylations in the normal rat hippocampus. Front Cell Neurosci 2019; 13: 179.

38. Salemi M, Cantone M, Salluzzo MG, Giambirtone M, Spada R, Ferri R. Reduced mitochondrial mRNA expression in dementia with Lewy bodies. J Neurol Sci 2017; 380: 122-3.

39. Essawy SS, Tawfik MK, Korayem HE. Effects of adenosine receptor antagonists in MPTP mouse model of Parkinson's disease: mitochondrial DNA integrity. Arch Med Sci 2017; 13: 659-69.

40. Papazafiropoulou AK, Koros C, Melidonis A, Antonopoulos S. Diabetes and dementia - the two faces of Janus. Arch Med Sci 2020; 5: e186-97.

41. Wierzejska R. Can coffee consumption lower the risk of Alzheimer's disease and Parkinson's disease? A literature review. Arch Med Sci 2017; 13: 507-14.

42. Holper L, Ben-Shachar D, Mann JJ. Multivariate meta-analyses of mitochondrial complex I and IV in major depressive disorder, bipolar disorder, schizophrenia, Alzheimer disease, and Parkinson disease. Neuropsychopharmacology 2019; 44: 837-49.

43. Keeney PM, Xie J, Capaldi RA, Bennett JP. Parkinson's disease brain mitochondrial complex I has oxidatively damaged subunits and is functionally impaired and misassembled. J Neurosci 2006; 26: 5256-64.

44. David DC, Hauptmann S, Scherping I, et al. Proteomic and functional analyses reveal a mitochondrial dysfunction in P301L tau transgenic mice. J Biol Chem 2005; 280: 23802-14 